\title{
Mechanical Properties of Biomaterials Used in Total Hip and Knee Arthroplasty
}

\author{
Sarah Kenney ${ }^{\#}$ and Christian Garner ${ }^{\#}$
}

Stephenson School of Biomedical Engineering, University of Oklahoma, Norman OK 73071

\begin{abstract}
Total hip and knee arthroplasty (THA and TKA) represent two of the most successful operations in orthopedics. For a total hip or knee prosthesis to function successfully, it must transfer mechanical loads up to seven times the individual's body weight from the axial skeleton to the lower extremities with minimal friction and wear. This is achieved by constructing prostheses from integrated components of different mechanical properties: a shock-absorbing and low-friction interface between the native joint and implant; a harder and stronger piece supporting the deformable interface, and an anchor securing the implant and transferring loads to the native bone. As early as the 1960s, polymers such as ultra-high molecular weight polyethylene (UHMWPE) were known to perform successfully at the joint-implant interface. Both ceramics and metals have historically been used for the main support of the implant, although metals and especially titanium alloys have taken preference in recent years. Recently, a flood of innovations has allowed material scientists to maximize the mechanical properties of these materials to increase mechanical strength, adjust elasticity, and improve biocompatibility. These innovations include the development of metal alloys and ceramic composites, reinforcement with carbon nanotubes and hydroxyapatite, antioxidant doping, gamma radiation-induced crosslinking, and bioactive coatings. Today, not only the mechanical properties but also the wear resistance and osseointegration of total hip and knee implants are far improved. This has led to better mechanical and physiological integration of the implants with the body and the necessary durability for younger patients' more active lifestyles. In this paper, these innovations will be explored within
\end{abstract}


the framework of implant mechanics to provide a comparative assessment of current materials' mechanical capabilities, advantages, and disadvantages.

Keywords: Total hip arthroplasty, total knee arthroplasty, prosthesis, mechanics 


\section{Introduction}

\section{The Growing Market of Prosthetic Materials}

Throughout history, advances in prosthetics have been intertwined with advances in the fields of biomaterials and biomechanics. Whereas the general architecture of total hip and knee replacements has remained largely homogeneous since the early 20th century, the biomaterials used have radically evolved as understanding of joint mechanics has improved. As surgical knowledge and the field of material science have expanded, health care systems have seen a drastic rise in the incidence of joint replacements due to osteoarthritis and other chronic diseases associated with joint pain. Since these diseases necessitate the implantation of a load-bearing device able to sustain joint function, it is critical that physicians and prosthetists stay up to date on the mechanical performance of different biomaterials.

Total hip and knee replacements have seen a particularly large rise in occurrence. Joint replacement in the lower limbs, including total hip arthroplasty (THA) and total knee arthroplasty (TKA), is commonly performed to improve the functionality of load-bearing joints and increase patient mobility. Data from 2010 would suggest that in the United States, almost 7 million individuals are living with a total knee replacement (Kremers et al., 2015), with the numbers for total hip replacements close behind (Pivec et al., 2012). Improved patient outcomes have prompted the expansion of THA and TKA, with much of this success owed to the development of innovative biomaterials that are more durable, withstand greater force, and are more cost-effective. More importantly, prosthetists are now able to customize implants to meet the specific needs of each patient. 
For this reason, it is imperative that physicians and prosthetists alike understand the mechanical performance of the wide range of biomaterials currently available for total joint arthroplasty and can evaluate these materials in the context of joint mechanics. The Society for Biomaterials lists over 65 biomaterials companies within the United States alone, and that count is expected to increase in coming years (SFB, 2014). There is a need to consolidate current information on the mechanical properties of both established and novel biomaterials.

TKA and THA share many properties, including materials used and load distribution. Because of this, the two will be discussed in conjunction throughout this paper. These implants have analogous components, serving similar functions in their respective joints, and these components can be discussed generally. For example, most hip and knee replacements utilize an articulation between the native and prosthetic joint surfaces to facilitate smooth movement. This articulation is of particular interest to prosthetists and has been the subject of numerous studies. The four classes of articulations most frequently used are metal-on-metal (MoM), ceramic-onceramic $(\mathrm{CoC})$, ceramic-on-polymer $(\mathrm{CoP})$, and metal-on-polymer (MoP). However, many researchers agree that the majority of TKA/THA implants utilize MoM and CoC articulations (Affatato, 2009). These types of articulations have been studied extensively and found to be the most structurally sound (Nabrdalik \& Sobociński, 2020) as well as best at suppressing immune response (Kenny et al., 2017).

\section{Common Causes of Arthroplasty Failure and Complications}

The current data on TKA/THA failures shows similar trends between the two procedures. Fatigue-resistant solutions have extended long-term survivability past 20 years to over $80 \%$ of THA implants (Pivec et al., 2012). TKA implants have nearly equal longevity but exhibit a larger incidence of revisional surgeries during early stages of implantation (Lum et al., 2018). 
Arthroplasty complications are becoming an increasingly rare occurrence but can still be fatal if left untreated.

Revisional surgeries are performed to correct problems with implants such as mechanical loosening of the anchors, instability, and dislocations. Combined, these three etiologies comprise $42 \%$ of THA failures (Pivec et al., 2012; Delaunay et al., 2013) and 46\% of TKA failures (Lum et al., 2018). There is not a singular cause for these occurrences; compounding factors including patient rehabilitation, surgical complications, and the response of the bone to the foreign material (Delaunay et al., 2013). It is already recognized that bone mass and volume decrease with age, and this change in structure can exacerbate failures (Khoo, 2016).

Furthermore, ill-fitting prosthetics can trigger more serious problems if revisional surgery is not performed. A common concern of early prosthetists was the risk of osteolysis and subsequent periprosthetic fracture (Delaunay et al., 2013). This complex syndrome is the result of the body's immune system rejecting a foreign material, which leads to excessive inflammation, irregular bone growth, unnatural load dispersion, and possible fractures along the bone-prosthetic interface (Ribeiro-da-Silva et al., 2018). As researchers have synthesized more biocompatible materials, the incidence of osteolysis and periprosthetic fracture has dropped considerably. Complications due to these events now account for 13\% of THA failures (Pivec et al., 2012) and 17\% of TKA failures (Lum et al., 2018). While our knowledge of the foreign body reaction has decreased their occurrence, they remain one of the deadliest failures of total arthroplasties.

\section{Mechanical Properties and Testing of Implant Materials}

As mentioned above, the number of available biomaterials grows each year. A prosthetist researching the most suitable material for an implant must evaluate the mechanical properties and 
biocompatibility of all materials on the market. One goal of this paper is to assist prosthetists, researchers, and physicians by consolidating this information into a single resource.

Teoh (2000) compiled a list of prosthetic materials including various metals, ceramics, and polymers used at the time. While the list was not comprehensive, it provided the groundwork for how biomaterials are evaluated in the context of prosthetics today. It identified three approaches to testing materials using simulated physiologic multiaxial loading that account for both acute forces and material longevity: (1) stress/life ( $\mathrm{S} / \mathrm{N})$ approach, (2) fracture mechanics approach, and (3) fatigue-wear approach (Teoh, 2000). The first two approaches examine mechanical properties when a material is at the verge of failure (e.g. fracture strength and yield strength), while the third quantifies material response to sustained pressure from multiple directions, mimicking the environment of native joints. This article primarily uses the first two approaches as a guide for evaluating the mechanical properties relevant to total hip and knee prostheses. The third approach is more relevant in computer simulated studies, whereas our sources contain mostly observationally derived data from real-world studies. Thus, it is not frequently considered in this particular article.

\section{Background}

\section{Summary of Key Terms}

A number of key biomechanical parameters will be helpful to define at the onset of this paper. In order to comparatively evaluate the performance of biomaterials in hip and knee prostheses, mechanical properties such as yield strength, rate of wear, and fatigue resistance will be explored. To discuss these, stress and strain must first be defined. Stress in biomechanics is defined as the instantaneous force applied to an object divided by the object's cross-sectional area. It is qualified in terms of strength. Yield strength refers to the amount of stress at which a material 
transitions from reversible to irreversible deformation, as compared to ultimate tensile strength, which refers to the maximum stress endured by a material before fracture. Fracture toughness describes the resistance of a material to fracture after a flaw has developed and is important in materials engineering for determining the risk of material failure after a crack appears. Calculating fracture toughness requires the calculation of stress intensity, or the amount of stress at the tip of a crack. Fracture toughness is the maximum stress intensity a material can withstand in a certain position without compromising its integrity. These parameters are critical in the evaluation of mechanical performance, particularly as it regards joint implants, which undergo large multiaxial stresses and are not ideal to replace if cracks develop. Fatigue failure, on the other hand, is a type of subsurface material compromise that occurs due to cyclic loading, a term for repetitive application of stress at a certain location. Fatigue resistance is a commonly used parameter when analyzing the performance of welded components in situations of repetitive stress, such as occurs at the point of stem fixation in artificial hip implants (A. Westerman et al., 2018), which will be discussed in more detail later in this paper.

The type of deformation undergone by a material is a qualification of strain, which is defined as the natural logarithm of the current length of a material divided by its initial length. Deformation can be either elastic or plastic. Plastic deformation is irreversible and occurs only after yield strength has been reached. Elastic deformation is reversible and characterized by Young's modulus of elasticity, which equals the ratio of stress to strain during elastic deformation. Young's modulus is also useful for describing the brittleness or ductility of a material. A brittle material undergoes a large amount of stress at minimum deformation and therefore has a high Young's modulus. A ductile material undergoes a large amount of strain when a small force is applied and therefore has a low Young's modulus. Both types of material may be either strong or 
weak, with strong referring to the ability to withstand high amounts of stress before irreversible deformation and weak referring to the occurrence of irreversible deformation at low amounts of stress. If the source of stress is in motion, the stress is described as a dynamic load; stationary sources of stress are known as static loads. For ideal load distribution, knee prostheses are designed to mimic femoral roll back, the rotation of the knee as the tibia exhibits anterior translation relative to the femur (L. Honório de Carvalho et al., 2011).

Wear is another critical component of biomaterials evaluation, describing surface damage to an implant from mechanical stress that causes debris release and contributes to osteolysis (D. Bitar et al., 2015). Osteolysis causes implant loosening, the primary cause of arthroplasty failure (D. Bitar et al., 2015); therefore, it is desirable that materials exhibit wear resistance. The primary mechanical factor controlling wear behavior is the degree of cross-linking in the material (M. Merola et al., 2019). Cross-linking is bonding between polymer chains that directly correlates to mechanical strength, resistance to abrasion, solvent resistance, and with certain methods, resistance to oxidation (Lubrizol, 2019; O. Muratoglu et al., 2001). It is closely related to crystallinity, which is the degree of order characterizing polymer chain orientation. A very ordered structure is highly crystalline, whereas a structure composed of randomly oriented chains has low crystallinity and is highly amorphous.

\section{Structure of Hip Prosthesis}

Four key components comprise a hip prosthesis, as shown in Figure 1. A metal acetabular shell fits inside the hip socket. Inside the shell is a plastic liner that functions as a weight-bearing interface; this layer must be both smooth and highly wear-resistant because of the chronic friction it experiences during user movement. Affixed to the liner and capping the femoral stem is the artificial femoral head. In a ball-and-socket joint, the femoral head is equivalent to the ball and is 
the center of the transverse, longitudinal, and sagittal axes about which the joint rotates (M. Gold et al., 2020). Inserted into the femoral medullary shaft is the femoral stem, a metal pin that stabilizes the prosthesis (A. Jahan et al., 2016).

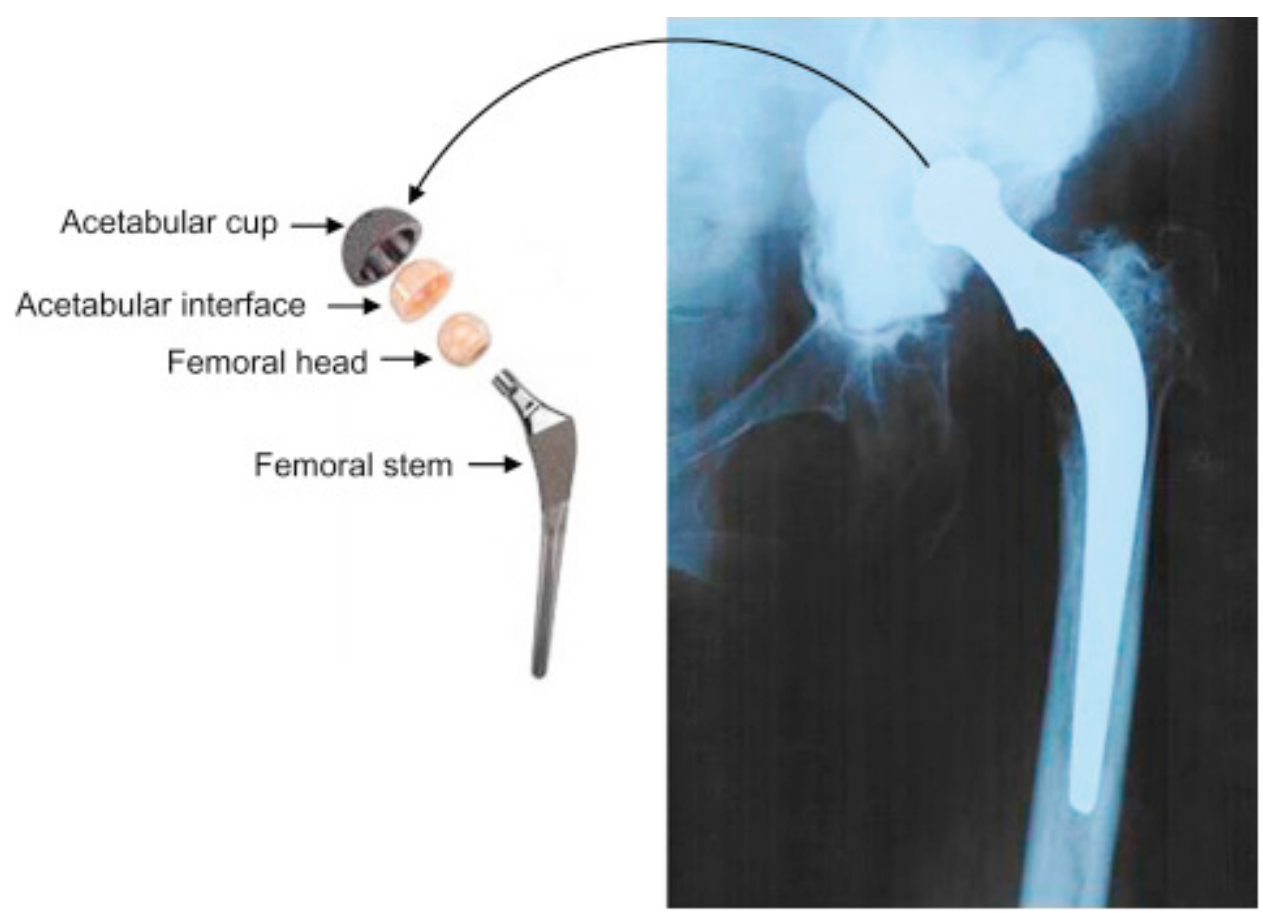

Figure 1. Diagram and X-ray of a hip prosthesis outlining four main components: an acetabular shell, acetabular interface, femoral head, and femoral stem. (A. Jahan et al., 2016)

\section{Structure of Knee Prosthesis}

Four key components comprise a knee prosthesis. The femoral component is a smooth metal piece with grooves imitating the femoral condyles that serves to resurface the worn lower end of the femur, as shown in Figure 2 (P. Manner, 2016). Imitating natural cartilage, a plastic spacer cushions the femoral component against the tibial component, reducing friction at the hinge (P. Manner, 2016). The tibial component, a curved metal plate similar to that used for the femur, resurfaces the worn portion of the upper tibia (P. Manner, 2016). On the backside of the kneecap, which is not visible in Figure 2, a dome-shaped artificial ligament made of strong plastic is used to replace the patella (C. Ma et al., 2018). 


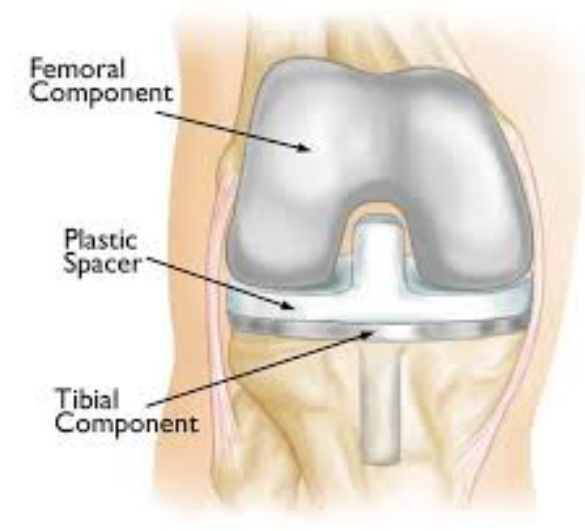

Figure 2. Diagram of a knee prosthesis outlining three key structures: a femoral component, plastic spacer, and tibial component (P. Manner, 2016).

\section{History of Hip and Knee Prostheses}

The history of hip and knee prostheses extends back to the 19th century. The first recorded attempt at hip arthroplasty is that of Dr. Anthony White, who in 1821 performed an excision arthroplasty and succeeded in reducing pain and improving mobility at the joint (P. Gomez et al., 2005). However, the repaired joint was highly unstable. In 1840, the medical field began exploring the first hip prostheses and even a crude version of the modern approach, resurfacing the femoral head with a wooden block (M. Merola et al., 2019). Since wood is not biocompatible, enormous wear debris was generated at the joint and the implant quickly failed (M. Merola et al., 2019). This sparked an exploration of biocompatibility and led to similar resurfacing attempts in the hip and knee with softer tissues, including human skin, pig bladder, adipose tissue, and gold foil (M. Merola et al., 2019; C. Dall'Oca et al., 2017).

Known as the first official hip and knee prostheses, fixated ivory ball-and-socket and hinge implants were developed by Dr. Themistocles Glück of Germany in 1890 (D. Bitar et al., 2015). The fixation of these implants with nickel-plated screws (P. Gomez et al., 2005) improved upon earlier interpositioning of soft tissue, which was unattached. Realizing that large ivory implants 
were rejected by the body, Dr. Glück began using a mixture of plaster of Paris, powdered pumice, and resin (M. Merola et al., 2019). In 1925, American surgeon Marius Smith-Petersen invented mold arthroplasty, the method of molding a hollow hemisphere onto the femoral head to facilitate smooth interaction at the acetabular interface (M. Merola et al., 2019). His model used glass and Bakelite, which proved too fragile for a location of such high stress (P. Gomez et al., 2005). However, mold arthroplasties using Vitallium, an electrically inert alloy of cobalt, chromium, and molybdenum (D. Bitar et al., 2015), exhibited promising clinical results and became the first reliable implant for hip replacement (P. Gomez et al., 2005).

In 1938, Dr. Philip Wiles pioneered the THA, using a stainless steel implant affixed to the bone with bolts and screws (M. Merola et al., 2019). This was shortly followed by Drs. Austin Moore and Harold Bohlman, who in 1940 used Vitallium in the first femoral stem implant, a structure that has persevered into modern THA (D. Bitar et al., 2015). In 1951, Dr. Kenneth McKee of England became the first orthopedic surgeon to perform THA with MoM prostheses, replacing both the acetabular cup and femoral head (D. Bitar et al., 2015). Similar to many of his predecessors, he encountered a high rate of failure due to implant loosening (P. Gomez et al., 2005). The 1950s also saw the advent of the hinged prosthesis for total knee replacement, pioneered by Dr. Börje Walldius of Sweden (A. Hawk, 2018). Various modifications of the total knee replacement followed in rapid succession, such as an acrylic plateau for the tibial head that improved stability and metallic plates that improved results for rheumatoid arthritis patients (C. Dall'Oca et al., 2017).

The innovations of Moore, Bohlman, and McKee paved the way for the work of the orthopedic surgeon who has come to be known as the "Father of Modern Arthroplasty", Sir John Charnley, who revolutionized hip implants in the 1960s (D. Bitar et al., 2015). Sir Charnley utilized 
a polymer acetabular cup, which significantly reduced friction at the acetabular interface by means of hard-soft coupling, a stainless steel femoral stem, and poly-(methyl methacrylate) (PMMA) to affix the implant to the bone (M. Merola et al., 2019; E. Kaivosoja et al., 2013). After recording significant wear and insufficient load-bearing capacity from a polytetrafluoroethylene (PTFE) cap, he began using high-density polyethylene (HDPE) and ultra-high molecular weight polyethylene (UHMWPE), with significant improvements (M. Merola et al., 2019). Having achieved a lowfriction, high load-bearing design, innovation in THA shifted to increasing the durability of total joint replacements and decreasing aseptic loosening (D. Bitar et al., 2015). In this way, Sir Charnley enabled the field of THA to make rapid strides in longevity and biocompatibility, producing what is now considered one of the most successful biomedical implants.

Likewise, the late 1960s and 1970s witnessed an onslaught of innovation in TKA initiated by the contributions of the French consortium GUEPAR (A. Hawk, 2018). GUEPAR developed an offset hinge prosthesis, a solid chrome-cobalt-molybdenum alloy rod that rigidly connected the femur and tibia but lacked an impact-absorbing material akin to the meniscus (A. Hawk, 2018). Although it exhibited a $73.5 \%$ failure rate within 5 years, the GUEPAR design paved the way for exponential innovation. In 1974 the first total condylar prosthesis (TCP) was developed, a nonhinged implant that utilized a chrome-cobalt femoral shield and polyethylene patellar and tibial components to reconstruct the joint (C. Dall'Oca et al., 2017). This improved weight distribution and stability during flexion, but did not resolve femoral anterior translation during flexion and produced significant wear debris (C. Dall'Oca et al., 2017). In 1978, Dr. John Insall of New York identified key limitations of the GUEPAR and TCP prostheses and developed the posteriorstabilized total knee prosthesis in response, with significant improvements including improved stability during flexion (A. Hawk, 2018). Since then, improvements such as improved posterior 
stability, eliminating the need to excise the cruciate ligament, use of a mobile meniscal component to improve knee rotation, and variations of the rigid hinge model that improve axial motion have characterized the field of TKA prostheses (C. Dall'Oca et al., 2017).

\section{Recent Advances}

\section{Selection Parameters}

Currently, the most common materials used in hip and knee prostheses are metals such as titanium and cobalt-chromium alloys, ceramics such as alumina and zirconia, although more frequently composites of the two; and polymers such as UHMWPE and high-crosslinked polyethylene (XLPE). In selecting materials for different prosthesis components, engineers evaluate the similarity of a material's mechanical properties to that of the component's anatomical counterpart. For example, modulus matching plays a significant role in the selection of materials for the femoral stem. If the modulus of elasticity of the synthetic femoral stem surpasses that of the femur, which at its cortical bone segments has been estimated at 10-30 GPa (Kunii et al., 2019), the implant's greater stiffness leads to upper-body loads being transferred disproportionately to the implant rather than shared between the femur and prosthesis (Choroszyński et al., 2017). This results in a biomechanical phenomenon known as stress shielding, in which decreased loadbearing triggers severe cortical bone resorption at the proximal end of the femur (Choroszyński et al., 2017). Stress shielding is a primary cause of aseptic loosening and implant failure (Luo et al., 2020). Therefore, to avoid damage to the femur and protect against implant loosening, it is critical to select an implant material with a similar modulus of elasticity. The best-fit materials per this requirement will be examined in the following sections. 


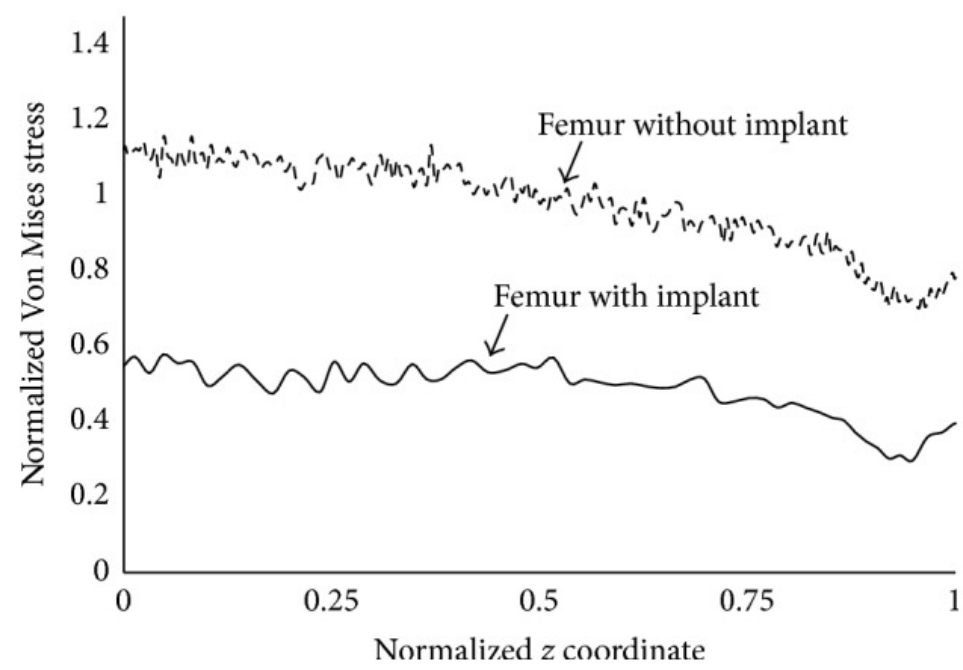

Figure 3. Effect of stress shielding on the femur (Ait Moussa et al., 2017). Since the drop in stress is largest at the proximal end of the femur $(z=0)$, the cortical bone here is most susceptible to resorption.

A second key criterion is the ultimate tensile strength of materials used for the acetabulum, femoral head, and femoral stem, which must be greater than the upper-body loads transferred at the hip in order to prevent implant fracture. Uniaxial tensile testing can be performed to measure tensile strength. Studies have indicated that the site of highest tensile stress, maximum bending moment, and most frequent fracture initiation in titanium femoral stems is the anterior lateral aspect at the component-cement interface (Westerman et al., 2018). This suggests that understanding not only the tensile strength but the flexural strength of a material is critical to addressing implant failure modes. Ultimate flexural strength can be measured using three- or fourpoint bending tests and has been determined to be highest in metals, followed by ceramic composites, and lowest in polymers - a trend identical to that for tensile strength (Rocha et al., 2006; Moraes et al., 2004; MatWeb). This demonstrates two mechanical parameters contributing to the selection of metals, alloys, and ceramics rather than polymers for construction of the femoral stem.

Related to the examination of tensile and flexural strength at the most vulnerable point on the hip prosthesis is the examination of material resistance to fatigue and fracture. International 
standards for pre-clinical fatigue performance testing require a fatigue safety factor, or a multiple of the load-bearing requirements on the implant, to be withstood during testing in order to increase the durability and therefore safety of the device (Westerman et al., 2018). Fatigue performance tests consist of cyclic compressive loading on the device for a number of cycles equivalent to its duration of use. This is important for the simulation of material behavior at sites where such cyclic loading occurs in vivo. This type of load is applied at the point of stem fixation in hip implants due to the cyclical muscle contraction and relaxation of the hamstrings and quadriceps during everyday activities such as walking, running, and going up stairs, with high effective body weights experienced at the lower limb joints due to the pounding action associated with such movements. For example, a standard walking pace causes approximately five times one's body weight to be loaded on the hip and nearly three times one's weight on the knee; going up or down stairs is associated with a more than sevenfold increase in effective body weight at the hip and more than fourfold at the knee (Geringer et al., 2013). These multiples of effective body weight must be accounted for when evaluating the fatigue resistance of different materials to construct the most suitable prosthesis for a given patient.

Given that a material meets the mechanical demands on a prosthesis, wear resistance reflects whether it is safe for long-term implantation. Wear resistance is often measured simultaneously with friction characteristics using a multiaxial hip simulator, which evaluates the performance of the entire prosthesis during simulated walking cycles (R. Sonntag et al., 2017). Knowledge about friction characteristics provides information on the dynamic force profile of the joint, such as net torque and the oscillation of extension-flexion cycles (R. Sonntag et al., 2017). However, it is most applicable in selecting a material to mimic articular cartilage, which reduces friction and distributes stresses at the hip. 


\section{Metals and Alloys}

This section will highlight the most relevant characteristics of metals commonly used in THA and TKA. Useful properties include the mechanical profile, biocompatibility, and incidence of failure.

\section{Stainless Steel}

Stainless steel is a common metallic alloy made from an iron-chromium mixture. Traditionally, biomedical devices using stainless steel also contained nickel, but nickel-free stainless steel has greatly improved corrosion resistance (Prasad et al., 2017). One type of lowcarbon stainless steel that is widely used in implants is $316 \mathrm{~L}$ stainless steel. Studies show that $316 \mathrm{~L}$ stainless steel has a yield strength of $190 \mathrm{MPa}$ and ultimate tensile strength of 490MPa (Hendra et al., 2011), making it an ideal candidate for physiological load-bearing. With in vivo testing, stainless steel implants have been noted to "produce longer-term changes in blood composition" (Eliaz, 2019), particularly regarding the concentration of metal ions such as chromium and nickel. Although still occasionally used, most THA/TKA MoM implants now use alloys with more favorable immune responses.

\section{Cobalt-Chromium Alloys}

Cobalt-chromium (Co-Cr) alloys are an attractive alternative to stainless steel and began seeing widespread use in the early 21 st century. Their increased wear resistance relative to stainless steel implants (Prasad et al., 2017) and significantly improved mechanical properties (Hendra et al., 2011) have made Co-Cr alloys one of the most utilized materials for total hip and knee replacements. A common Co-Cr alloy product, CoCrWNi (F90), has a yield strength higher than that of stainless steel at $310 \mathrm{MPa}$ and an ultimate tensile strength of $860 \mathrm{MPa}$ (Hendra et al., 2011; Eliaz, 2019). It has been noted that long-term wearers of cobalt-chromium implants show elevated 
blood levels of metallic ions including hexavalent chromium, trivalent chromium, and cobalt ions, a mix of potential and known carcinogens (Cohen, 2012). Nevertheless, cobalt-chromium alloys remain a popular choice for biomaterial scientists.

\section{Titanium Alloys}

Titanium alloys are among the newer biomaterials on the market and have gained popularity due to their reduced weight and high mechanical strength. Newer titanium-based alloys, such as Ti33Zr5Fe4Cr, show a yield strength of over $1210 \mathrm{MPa}$ and an ultimate tensile strength of $1711 \mathrm{MPa}$ (Liang-Yu et al., 2020); however, they have limited clinical application due to their novelty. More traditional titanium alloys, such as Ti6A14V (F136), still show greater strength than Co-Cr implants (Hendra et al., 2011). In terms of biocompatibility, titanium is more favorable than stainless steel or Co-Cr THA/TKA implants since it displays only minimal release of metallic ions, leading many researchers to deem it a bioinert material (Eliaz, 2019).

\section{Titanium Alloy Surface Modifications}

Titanium alloys show remarkable biocompatibility and mechanical properties, and biomaterial scientists are able to enhance these qualities through surface modifications. Increasing the porosity of titanium alloys has been shown to stimulate bone growth in orthopedic implants and reduce the Young's modulus to a more physiologically imitative range (Kirmanidou et al., 2016). Various surface modifications have been attempted to amplify the mechanical properties of titanium alloys, namely anodic oxidation and chemical vapor deposition, both of which aid in decreasing wear and corrosion of the material (Kirmanidou et al., 2016).

\section{Ceramics}

Ceramics are a unique class of biomaterials generally characterized by brittleness and rigidity. Although early ceramics were simple ionic compounds containing a metal and a non- 
metal, material scientists have maximized their mechanical advantages and created a new field of complex biomaterials to be studied. Today, many THA/TKA implants use CoC or CoM articulations to reduce corrosion due to wear. This section will discuss popular ceramics for hip and knee replacements, used either as coatings or bulk materials.

\section{Novamin/Bioglass}

Bioglass is a bioactive ceramic material largely composed of calcium, sodium and phosphorus. Commonly used in orthopedic and dental applications, bioglass has been established since the 1990's as a suitable material for hard tissue replacement. Its composition and mechanical properties are very similar to teeth and thus it is often used as a topical treatment for enamel wear (Vahid et al., 2012). In the case of contemporary orthopedic implants, including TKA and THA, bioglass and its derivative Novamin are commonly used as a coating for metallic bearings (Sergi et al., 2020), as opposed to a bulk material. Due to its similarities to bone, bioglass shows excellent biocompatibility. In fact, So et al. showed that patients with bioglass-coated THA implants had a 100\% survivability rate at 9 years post-operation (2012), demonstrating the long-term compatibility of this material.

\section{Alumina}

Alumina is another ceramic commonly used as a coating for metallic bearings in TKA/THA implants. Although alumina traditionally shows good wear resistance, there is a higher incidence of fracture within the first 15 years after implantation than other ceramics (Chang \& Taek-Rim, 2018). Since alumina is not very bioactive in orthopedic implants, it is often used in conjunction with a bioglass ceramic (Georgiana-Alexandra et al., 2020) or zirconia (Merola \& Affatato, 2019) to inhibit osteolysis. It is rare to find a pure alumina coating; however, even when applied as a thin film, alumina improves an implant's mechanical properties, making it a desirable prosthetic material.

\section{Zirconia}

Like alumina, zirconia is rarely used by itself as a coating but rather is supplemented with bioglass, alumina, or a combination of the three (Baino et al., 2019; So et al., 2019; Georgiana-Alexandra, 2020). In 
comparison to alumina and bioglass, zirconia shows higher malleability and a lower ultimate tensile strength (Baino et al., 2019). Zirconia has the benefit of being more bioactive than alumina (Sergi et al., 2020), and often the two are used together in orthopedic implants to improve strength and biocompatibility (Chang \& Taek-Rim, 2018).

\section{Polymers}

Since the 1960s, polymers and particularly UHMWPE have remained the primary material for artificial bearing surfaces at the hip and knee joints. A polymer interface between harder ceramics or metals used for the acetabulum and femoral head exhibits hard-on-soft coupling, a beneficial phenomenon associated with reduced friction and wear at the interface (Affatato et al., 2018). Another common polymer in TKA/THA is PMMA, also termed bone cement, which is used to affix the femoral stem in the medullary cavity as well as repair small bone defects in revision TKA (Hasandoost et al., 2020). The polyethylenes of Sir John Charnley's era have evolved considerably with innovations in this decade aimed at improving wear resistance, such as antioxidant doping and gamma radiation-induced crosslinking.

\section{Modulus of Elasticity}

Given the extremely low modulus of elasticity of articular cartilage, $0.0038-0.016 \mathrm{GPa}$, it is critical that imitative materials demonstrate similar flexibility. Standard polyethylene has a modulus of elasticity of $0.88 \mathrm{GPa}$, which quite nearly approaches this target (Ghalme et al., 2016). High-density polyethylene (HDPE) has a reported modulus of elasticity of 0.565 - $1.50 \mathrm{GPa}$ and an average elongation at yield of $10.8 \%$, indicating high flexibility and ductility (MatWeb). UHMWPE has a reported modulus of elasticity of $101 \mathrm{GPa}$; this mismatch contributes to chronic wear particle release, a significant impairment to the prosthesis and one that has been met with a variety of innovative improvements (Werff and Heisserer, 2016). One such innovation is reinforcement with multi-walled carbon nanotubes to increase UHMWPE ductility up to $140 \%$ (Ghalme et al., 2016).

\section{Tensile Strength}

While low modulus of elasticity is necessary, polymers are unique among biomaterials in that they can maintain this property without compromising tensile strength, another important requirement of the 
acetabular interface. The tensile strength of standard polyethylene is reported at $35 \mathrm{MPa}$, whereas UHMWPE raises this metric to around $52 \mathrm{MPa}$ (Ghalme et al., 2016; Gigante et al., 2015). XLPE exhibits poorer tensile strength than UHMWPE but improved wear resistance (Merola, 2019). Since in vitro estimates of the contact stress on articular cartilage fall around 0.5-5 $\mathrm{MPa}$, each of these polymers is sufficiently strong to fulfill the role of the acetabular interface (Brand, 2005).

\section{Wear Resistance}

The primary determinant of polymer performance at the acetabular interface and in the femoral cavity, in the case of cemented implants, is wear resistance. Although polyethylene is notorious for its poor wear resistance, modifications such as Vitamin E (VE) doping of UHMWPE appear promising. This works by either adding VE to the powdered polymer, then irradiating the consolidated blend to cause cross-linking, or by diffusing the radiation-treated polymer with VE and then sterilizing it with gamma rays (Ghalme et al., 2016). The biological antioxidant terminates free radical chain reactions by donating a hydrogen from its $\alpha$-tocopherol group to stabilize free radicals. This prevents oxidative degradation of polymer chains, promotes the longevity of the implant, and protects the surrounding bone from osteolysis. A study on subcutaneous implantation of VE-doped UHMWPE in rats found no oxidation of UHMWPE films after a six month period, underscoring the promise of this method (Ghalme et. al, 2016). Using hip simulators, the wear performance of this polymer has been evaluated and found to be improved upon conventional UHMWPE without corresponding changes of tribological properties (Ghalme et al., 2016).

Increasing the degree of crosslinking of polyethylene has also been shown to improve wear resistance of hip prostheses (Bracco et al., 2017). Analyzing the wear mechanism of UHMWPE, researchers in the late 20th century noted that wear predominantly occurred during plastic deformation: polymer molecules align in the direction of contact forces, the parallel fibrils undergo strain and are elongated, the ultimate tensile strength decreases, and the modulus of elasticity increases such that the material becomes more brittle (Bracco et al., 2017). The weakening of the material facilitates the loss of wear particles. Crosslinking was developed to slow such deformation by increasing the amount of alkene bonds in the polymer backbone, thereby decreasing its molecular flexibility. 
As opposed to VE doping, cross-linking requires free radical formation along the polymer backbone to form carbon-to-carbon covalent bonds, or crosslinks (Ghalme, 2016). This can be achieved with a few different methods of radiation; the best results have been achieved with gamma radiation, thermal treatment above the melting temperature of the polymer, and anaerobic sterilization $(\mathrm{Hu} \&$ Yoon, 2018). Thermal treatment melts crystalline molecules, terminating any crystalline-phase free radicals and improving the oxidative stability of the crosslinked polymer (Bracco et al., 2017). Studies on the wear performance of XLPE in THA have reported $42 \%$ to $95 \%$ reduction in wear compared to conventional polyethylene and significant decreases in osteolysis (Hu \& Yoon, 2018). However, the fatigue resistance and mechanical strength is significantly lower than that of VE-doped UHMWPE (Gigante et al., 2015).

\section{Polymer Composites}

Recently the benefits of poly-2-methacryloyloxyethyl phosphorylcholine (PMPC) grafting with other polymers to achieve a composite of the strength and wear resistance of two materials have become apparent. Carbon fiber-reinforced poly-ether-ether-ketone (CFR-PEEK) is a polymer demonstrated to have excellent mechanical strength but poor wear resistance at the acetabular interface, with a THA survival rate of only $86.5 \%$ in a case study of 29 THA patients (Heijnens et al., 2020). However, acetabular interface liners constructed from PMPC grafted withhu CFR-PEEK have achieved improved wear rates in hip simulator testing compared to liners constructed from untreated CFR-PEEK (Yamane et al., 2018). Additionally, PMPC grafting with XLPE has been shown to produce a super-lubricious surface that reduces wear by up to 70 million cycles in hip simulator testing (Hu \& Yoon, 2018). Such polymer composites may be the key to achieving the ideal combination of cartilage-like wear resistance and tensile strength for a longer-lasting acetabular interface.

\section{Biocomposite Materials}

A biocomposite material is an umbrella term for biomaterials that include a mixture of ceramics,

metals, and polymers. Most biocomposite materials comprise a polymer and a supplemental, structurally supportive material. This includes a wide range of materials and is a novel field in prosthetic engineering.

\section{Polyetheretherketone (PEEK)}


PEEK is a thermoplastic polymer used in several biomedical applications, such as prosthetics and tissue engineering. By itself, PEEK is bioinert (Panayotov et al., 2016); however, when combined with hydroxyapatite (HA), it shows increased bioactivity and greater tensile strength (Ma et al., 2014). Furthermore, often a bioactive solute is required as native PEEK has been shown to exhibit "poor osseointegration" (Evans, 2015). It is likely that this is one reason PEEK is typically used as an articulation covering in TKA/THA prosthetics (Panayotov et al., 2016), similar to what is seen with implant ceramics. It has been noted that PEEK caps on the ends of metallic bearings in hip replacements show a quicker rate of wear and debris generation than ceramic caps alone (Panayotov et al., 2016).

\section{UHMWPE-Carbon Fibers}

UHMWPE is one of the most common polymers for TKA/THA implants. Consistent with polymer behavior, UHMWPE shows viscoelastic properties under acute stress (Deng \& Uhrich, 2010). While viscoelasticity is an important characteristic of native tissue, it is beneficial to reduce this property in implanted materials so as to decrease internal stresses faced by the implant during loading and unloading at the joint. Fully elastic materials will limit the amount of energy dissipated to the surrounding tissue with each loading cycle. Integrating carbon nanotubes into the UHMWPE during solidification of the polymer has been shown to reduce this viscoelasticity, particularly reducing creep resistance (Ghalme et al., 2016). This is a novel area of research, taking cues from architectural and structural engineering motifs to improve the mechanical properties of a material without sacrificing its lightweight advantage - an equally important consideration in prosthetic design. Additionally, graphene oxide, a carbon-based derivative, has shown promise in reducing wear debris in orthopedic implants (Moreno, 2013).

\section{Hydroxyapatite}

HA is a well-known and widely used composite material in many areas concerning bone regeneration. Many researchers utilize HA because of its excellent biocompatibility and the similarity between its mechanical properties and that of native bone. This is unsurprising, as hydroxyapatite is a natural component of bone tissue (Habibah et al., 2020). Used primarily as a coating for the femoral stem, HA has shown increased long-term survivability and osseointegration when applied to THA implants 
(Castellini et al., 2016). Additionally, one study has shown that TKA implants with any degree of HA coating were shown to have excellent long-term survivability in comparison to TKA implants without HA coating (Epinette \& Manley, 2007). In both TKA and THA implants, HA provides resistance to bone resorption (Habibah et al., 2020), increasing implant longevity.

\section{Comparison of Foreign Body Response at Bone-Implant Interface \\ Osseointegration in Metal Implants}

Osseointegration is a direct physical and functional connection of living bone with a prosthetic surface. It is a critical factor in the prevention of aseptic loosening, the primary reason for revision arthroplasties (Liu et al., 2020). Research has shown that highly porous materials stimulate osseointegration (Lewallen et al., 2015). Certain metals can be molded with over $65 \%$ porosity, including titanium, titanium alloys, and tantalum (Lewallen et al., 2015); this is promising for metal-on-metal hip prostheses. One study of periacetabular bone ingrowth observed $49.1 \pm 27.9 \%$ bone ingrowth for hydroxyapatite-coated Co-Crbeaded implants of ideal bead diameter, pore size, and porous volume (Mukherjee \& Gupta, 2017). Liu et al. draws the conclusion that implant surfaces made of materials that replicate bone structure and mechanical properties, such as hydroxyapatite, experience greater and more rapid osseointegration. Bone ingrowth not only increases the modulus of elasticity of the implant (Mukherjee \& Gupta, 2017), thus strengthening against fracture and fatigue failure, it also protects against stress shielding and aseptic loosening by incorporating the native bone with the implant. The protective, bioactive nature of such implants presents a strong advantage over untreated metals and alloys.

\section{Osseointegration in Ceramic Implants}

Although ceramics are structurally more similar to bone than are metals, their brittleness prohibits the same success in load-bearing (Brunello et al., 2019). However, bone ingrowth can also be observed in ceramic implants when they are coated with a harder, bioactive material. One in vivo study on bioceramic implantation in sheep found that covalent binding to phosphate molecules on one implant and $20 \mathrm{~nm}$ hydroxyapatite coating on another both produced strong osseointegration on the implant surface (Pobloth et al., 2019). Twelve weeks after operation, ultimate tensile strength of these bioactive implants was 
sevenfold that of the uncoated prostheses (Pobloth et al., 2019). Ceramics dip-coated in $2 \mu \mathrm{m}$ of bioglass also demonstrated osseointegration, to a lesser degree. However, ceramic implants coated in a solid bioglass gel did not show the same success in osseointegration and even had lower moduli of elasticity than uncoated implants, which Pobloth et al. associated with a lympho-plasma cellular inflammatory reaction. Further studies are needed to evaluate the ability of bioglass coatings to stimulate osseointegration; however, this study reiterates the benefits of hydroxyapatite coating on ceramic and metal implants alike.

\section{Future Prospects}

\section{Shape Memory Materials}

The tendency of a material to conform to a specific shape after application of an external stimulus is known as the shape memory effect (SME), and materials that exhibit this phenomenon [shape memory materials: SMMs] are a relatively new field of study in biomaterial science (Huang, 2010). SMMs have been studied since the early 1900s but only entered into biomedical applications in the 1970s (Huang, 2010). There are two categories of SMMs commonly used in THA/TKA: shape memory alloys (SMA) and shape memory polymers (SMP). In both cases, SMMs do not constitute the bulk material itself, but rather act as braces or fixtures supporting the implant. Alloys that exhibit shape memory holding properties, particularly pseudoelasticity, are desirable due to their ability to more accurately mimic the native tissue's biomechanical properties, an advantage over more static and rigid metals (Petrini \& Migliavacca, 2011). Common SMA materials that are used in implants include alloys of nickel-titanium (NiTi), nickel-aluminum (NiAl), and silver-cadmium (AgCd) (Petrini \& Migliavacca, 2011). Shape memory polymers (SMP) are not as widely used as SMAs in orthopedic hard tissue implants, likely due to their reduced mechanical strength in comparison to most alloys. Typical SMPs include common biomaterials such as PLA and PCL, as well as novel materials such as poly(glycerol dodecanoate) (PGD) (Ramaraju, 2020). 
Researchers have seen some success in using SMAs to increase the initial stability of THA implants (Higa et al., 2010). Furthermore, during revisional THA, SMA materials have been used as a brace to help heal post-implantation periprosthetic fracture (Xiong et al., 2014). Although the materials used in SMA-based TKA implants are similar, the positioning of the alloy is slightly different. SMAs used in knee replacements are typically applied as spacers at the tibiofemoral joint and show fewer signs of long-term fatigue and deformation in comparison to standard polymer spacers (Gautam et al., 2018). The use of these materials could have implications for long-term, high stress prosthetics designed for more active lifestyles. The resiliency and tendency for a material to retake a specified shape is absolutely beneficial in THA/TKA implants, as this reduces the degree of wear a prosthesis experiences during load-bearing. These materials are an area of research deserving further exploration for prosthetists seeking to design an adaptable and longlasting implant.

\section{Antibiotic Delivery through Nanomaterials in Cemented Implants}

A burgeoning field of research within THA/TKA is the loading of PMMA with antibiotics incorporated into nanocomposite materials. The local release of antibiotics after operation serves to mitigate infection. This mechanism has many complications to be addressed, including coinfluential drug delivery profiles, decreased compressive strength and bending modulus of PMMA when mixed with antibiotics, and the difficulty of selecting a few antibiotics for defense against a large range of bacteria (Al Thaher et al., 2017). While this field has yet to achieve a mechanism for global defense against infection, significant advances have been made in retaining the mechanical properties of antibiotic-loaded PMMA using nanomaterials. Delivery with nanomaterials improves upon the traditional model of mixing powdered antibiotics with PMMA by providing a more even distribution of antibiotics and preventing the instantaneous post- 
implantation burst of drug release due to antibiotic surface accumulation (Ayre et al., 2015). Among the nanomaterials shown to deliver antibiotics without weakening the mechanical properties of PMMA are $15 \%$ by wt. chitosan nanoparticles, $7 \%$ by wt. propylparaben, and liposomes (Al Thaher et al., 2017; Ayre et al., 2015). Recently, researchers also tested novel combinations of crosslinked $\beta$-cyclodextrin $(\beta-C D)$ microparticles with various antibiotics and found that the $\beta-C D$ microparticles prevented mechanical weakening of the PMMA, while incorporating multiple antibiotics into the microparticles was associated with a longer antimicrobial effect (Cyphert et al., 2020). These innovations are very promising for improving patient outcomes following THA/TKA, as antibiotic-loaded nanomaterials integrated with PMMA can regularly sustain drug release over a month and in certain cases up to 70 days (Cyphert et al., 2020). Future research is necessary to quantify the load-bearing capability of PMMA integrated with antibiotic-laden nanocomposite materials before they can be integrated into THA/TKA (Cyphert et al., 2020). With further assessment of these materials' mechanical performance, the field of THA/TKA may witness an increase in the frequency of antibiotic loading of PMMA via nanocomposites and microparticles in upcoming years, potentially reducing rates of post-operative infection and revisional surgery.

\section{Conclusion}

Research into orthopedic implant materials is not a historically new field. However, with the explosion of innovations in polymer and alloy sciences, the focus of material research has shifted from simply searching for a suitable material to fine-tuning existing materials to attain the desired mechanical properties. Thus, with each year, the catalog of available materials for THA/TKA implants grows. The goal of most orthopedic implant material research is to minimize indices of failure rates and other in vivo complications. Long-term prosthetic survivability 
continues to climb each year; however, there is still room for improvement. Biocompatibility is one area that has received particular attention and is often the deciding factor in material selection. For example, the shift towards cobalt-chromium alloys, titanium-based alloys, and others that are known to leech fewer metallic ions represents a change in the attitude and direction of biomaterial science.

The number of TKA and THA procedures done each year is at an all-time high, confirming that these have become increasingly safe and effective operations. Novel materials such as SMMs and antibiotic-loaded nanocomposites are contributing to improved patient quality of life postimplantation. Metallic alloys such as cobalt-chromium continue to show dominance in the composition of most implants. Adding surface modifications, such as plasma-sprayed hydroxyapatite and ceramic coatings, has increased the wear resistance and structural integrity of these alloys. Additionally, polymer-based materials and polymeric coatings are commonly used modifications to decrease surface friction and more accurately mimic the elasticity of native tissue. These polymers are generally more bioinert than metals and alloys and degrade into less toxic constituents. However, this increased biocompatibility often comes at the cost of reduced mechanical strength. Finally, ceramic applications in THA/TKA implants are a well-established means of combining structural stability and biocompatibility. The scope that ceramics have for bioactivity is vast, ranging from almost completely bioinert (e.g. alumina) to being able to facilitate the growth of bone tissue (e.g. hydroxyapatite). Most ceramics in lower limb joint replacements appear in the articulations or as coating on a metallic alloy. The rigidity of most ceramics makes them less effective at being the main component of load-bearing prosthetics, as trauma to these areas would be more likely to cause fracture. 
The success of THA/TKA implants lies in a trifecta of three imperative characteristics: mechanical strength, elasticity, and biocompatibility. To reduce failure rates in joint replacements, prosthetists must design implants with a balance of these three characteristics. The combined use of metals, polymers, ceramics, or their various composites tends to produce prostheses with greater longevity and less incidence of failure. Novel materials must exhibit these qualities to contribute effectively to orthopedic prostheses. The field of total hip and knee replacements has undergone significant metamorphoses in approximating the mechanics of the native joints within recent years, largely thanks to nanotechnology enhancements, development of more biocompatible and bioactive materials, and a better understanding of the stresses involved at load-bearing joints.

\section{Authorship}

\# Sarah Kenney and Christian Garner contributed equally 


\section{References}

1. Ait Moussa, A., Fischer, J., Yadav, R., \& Khandaker, M. (2017). Minimizing Stress Shielding and Cement Damage in Cemented Femoral Component of a Hip Prosthesis through Computational Design Optimization. Advances in orthopedics, 2017, 8437956. https://doi.org/10.1155/2017/8437956

2. Affatato, S., Ruggiero, A., Jaber, S. A., Merola, M., \& Bracco, P. (2018). Wear Behaviours and Oxidation Effects on Different UHMWPE Acetabular Cups Using a Hip Joint Simulator. Materials (Basel, Switzerland), 11(3), 433. https://doi.org/10.3390/ma11030433

3. Affatato, S., Spinelli, M., Squarzoni, S., Traina, F., \& Toni, A. (2009). Mixing and matching in ceramic-on-metal hip arthroplasty: An in-vitro hip simulator study. Journal of Biomechanics, 42(15), 2439-2446. Doi: https://doi.org/https://doi.org/10.1016/j.jbiomech.2009.07.031

4. Al Thaher, Y., Perni, S., \& Prokopovich, P. (2017). Nano-carrier based drug delivery systems for sustained antimicrobial agent release from orthopaedic cementous material. Advances in Colloid and Interface Science, 249, 234-247. Doi: https://doi.org/10.1016/j.cis.2017.04.017

5. Ayre, W.N., Birchall, J.C., Evans, S.L., \& Denyer, S.P. (2016). A novel liposomal drug delivery system for PMMA bone cements. Journal of Biomedical Materials Research: Part B Applied Biomaterials, 104(8): 1510-1524. Doi: https://doi.org/10.1002/jbm.b.33488

6. Baino, F., Minguella-Canela, J., Korkusuz, F., Korkusuz, P., Kankılıç, B., María Ángeles Montealegre, .. . Vitale-Brovarone, C. (2019). In vitro assessment of bioactive glass coatings on Alumina/Zirconia composite implants for potential use in prosthetic applications. International Journal of Molecular Sciences, 20(3) doi:http://dx.doi.org/10.3390/ijms20030722

7. Bitar, D., \& Parvizi, J. (2015). Biological response to prosthetic debris. World Journal of Orthopedics, 6(2), 172-189. Doi: 10.5312

8. Bracco, P., Bellare, A., Bistolfi, A., \& Affatato, S. (2017). Ultra-High Molecular Weight Polyethylene: Influence of the Chemical, Physical and Mechanical Properties on the Wear $\begin{array}{lllll}\text { Behavior. A Review. Materials (Basel, } & \text { Switzerland), } & \text { 10(7), }\end{array}$ https://doi.org/10.3390/ma10070791

9. Brand R. A. (2005). Joint contact stress: a reasonable surrogate for biological processes?. The Iowa Orthopaedic Journal, 25, 82-94.

10. Brunello, G., Elsayed, H., \& Biasetto, L. (2019). Bioactive Glass and Silicate-Based Ceramic Coatings on Metallic Implants: Open Challenge or Outdated Topic? Materials (Basel, Switzerland), 12(18), 2929. https://doi.org/10.3390/ma12182929

11. Castellini, I., Andreani, L., Parchi, P. D., Bonicoli, E., Piolanti, N., Risoli, F., \& Lisanti, M. (2016). Hydroxyapatite in total hip arthroplasty. Our experience with a plasma spray porous titanium alloy/hydroxyapatite double-coated cementless stem. Clinical cases in mineral and bone metabolism : the official journal of the Italian Society of Osteoporosis, Mineral Metabolism, and Skeletal Diseases, 13(3), 221-227. https://doi.org/10.11138/ccmbm/2016.13.3.221

12. Chang, Y. H., \& Taek-Rim Yoon. (2018). Recent updates for biomaterials used in total hip arthroplasty. Biomaterials Research, 22 doi:http://dx.doi.org/10.1186/s40824-018-0144-8

13. Choroszyński, M., Choroszyński, M., \& Skrzypek, S. (2017). Biomaterials for hip implants Important considerations relating to the choice of materials. Bio-Algorithms and Med-Systems, 13(3), 133-145. Doi: 10.1515

14. Cohen, D. (2012). How safe are metal-on-metal hip implants?. BMJ : British Medical Journal (Online), 344 doi:http://dx.doi.org/10.1136/bmj.e1410

15. Cyphert, E.L., Chao-yi, L., Marques, D.W., Learn, G.D., \& von Recum, H.A. (2020). Combination Antibiotic Delivery in PMMA Provides Sustained Broad-Spectrum Antimicrobial Activity and Allows for Postimplantation Refilling. Biomacromolecules, 21, 854-866. Doi: 10.1021/acs.biomac.9b01523

16. Dall'Oca, C., Ricci, M., Vecchini, E., Giannini, N., Lamberti, D., Tromponi, C., \& Magnan, B. (2017). Evolution of TKA design. Acta Biomedica, 88(2), 17-31. Doi:10.23750 
17. Delaunay, C., Hamadouche, M., Girard, J., Duhamel, A., \& SoFCOT Group (2013). What are the causes for failures of primary hip arthroplasties in France?. Clinical orthopaedics and related research, 471(12), 3863-3869. https://doi.org/10.1007/s11999-013-2935-5

18. Deng, M., \& Uhrich, K. E. (2010). Viscoelastic behaviors of ultrahigh molecular weight polyethylene under three-point bending and indentation loading. Journal of biomaterials applications, 24(8), 713-732. https://doi.org/10.1177/0885328209102750

19. Eliaz N. (2019). Corrosion of Metallic Biomaterials: A Review. Materials (Basel, Switzerland), 12(3), 407. https://doi.org/10.3390/ma12030407

20. Epinette, J. A., \& Manley, M. T. (2007). Hydroxyapatite-coated total knee replacement: clinical experience at 10 to 15 years. The Journal of bone and joint surgery. British volume, 89(1), 34-38. https://doi.org/10.1302/0301-620X.89B1.17864

21. Evans, N. T., Torstrick, F. B., Lee, C. S. D., Dupont, K. M., Safranski, D. L., Chang, W. A., ... Gall, K. (2015). High-strength, surface-porous polyether-ether-ketone for load-bearing orthopedic implants. Acta Biomaterialia, 13, 159-167. https://doi.org/https://doi.org/10.1016/j.actbio.2014.11.030

22. Georgiana-Alexandra Prefac, Marina-Larisa Milea, Andreea-Mihaela Vadureanu, Muraru, S., Daniela-Ileana Dobrin, Gabriela-Olimpia Isopencu, . . . Busuioc, C. (2020). CeO0RW1S34RfeSDcfkexd09rT421RW1S34RfeSDcfkexd09rT4 containing thin films as bioactive coatings for orthopaedic implants. Coatings, $10(7), \quad 642$. doi:http://dx.doi.org/10.3390/coatings10070642

23. Geringer, J., Kim, K., Pellier, J., \& Macdonald, D.D. (2013). Fretting corrosion processes and wear mechanisms in medical implants. In Y. Yan (Ed.), Bio-Tribocorrosion in Biomaterials and Medical Implants. Woodhead Publishing. https://doi.org/10.1533/9780857098603.1.45.ghalme

24. Ghalme, S. G., Mankar, A., \& Bhalerao, Y. (2016). Biomaterials in Hip Joint Replacement. International Journal of Materials Science and Engineering, 4(2), 113-125. https://doi.org/: 10.17706/ijmse.2016.4.2.113-125

25. Gigante, A., Bottegoni, C., Ragone, V., \& Banci, L. (2015). Effectiveness of Vitamin-E-Doped Polyethylene in Joint Replacement: A Literature Review. Journal of Functional Biomaterials, 6(3), 889-900. https://doi.org/10.3390/jfb6030889

26. Gold, M., Munjal, A., \& Varacallo, M. (2020). Anatomy, Bony Pelvis and Lower Limb, Hip Joint. Retrieved October 19, 2020, from https://www.ncbi.nlm.nih.gov/books/NBK470555/

27. Gomez, P. F., \& Morcuende, J. A. (2005). Early Attempts at Hip Arthroplasty: 1700-1950s. Iowa Orthopaedic Journal, 25, 25-29. Retrieved October 19, 2020, from https://www.ncbi.nlm.nih.gov/pmc/articles/PMC1888777/.

28. Gotman, I. (1998). Characteristics of Metals Used in Implants. Journal of Endourology/Endourological Society, 11, 383-389. https://doi.org/10.1089/end.1997.11.383

29. Gautam, A., Callejas, M. A., Acharyya, A., \& Acharyya, S. G. (2018). Shape-memory-alloy-based smart knee spacer for total knee arthroplasty: 3D CAD modelling and a computational study. Medical engineering \& physics, 55, 43-51. https://doi.org/10.1016/j.medengphy.2018.03.001

30. Habibah T.U., Amlani D.V., Brizuela M. (2020). Hydroxyapatite Dental Material. StatPearls. Treasure Island (FL): StatPearls Publishing; 2020 Jan-. Available from: https://www.ncbi.nlm.nih.gov/books/NBK513314/

31. Hasandoost, L., Rodriguez, O., Alhalawani, A., Zalzal, P., Schemitsch, E.H., Waldman, S.D., Papini, M., and Towler, M.R. (2020). The Role of Poly(Methyl Methacrylate) in Management of Bone Loss and Infection in Revision Total Knee Arthroplasty: A Review. Journal of Functional Biomaterials, 11(2): 25. doi: 10.3390/jfb11020025

32. Hawk, A. J. (2018). ArtiFacts: The GUEPAR Offset Hinge Knee. Clinical Orthopaedics and Related Research, 476(4), 692-693. Doi: 10.1007

33. Heijnens, L. J., Schotanus, M. G., Verburg, A. D., \& van Haaren, E. H. (2020). Disappointing long-term outcome of THA with carbon-fiber-reinforced poly-ether-ether-ketone (CFR-PEEK) as 
acetabular insert liner: a prospective study with a mean follow-up of 14.3 years. HIP International. https://doi.org/10.1177/1120700020918157

34. Hendra Hermawan, Dadan Ramdan and Joy R. P. Djuansjah (2011). Metals for Biomedical Applications, Biomedical Engineering - From Theory to Applications, Prof. Reza Fazel (Ed.), ISBN: 978-953-307-637-9, InTech, Available from: http://www.intechopen.com/books/biomedical-engineering-from-theory-toapplications/metalsfor-biomedical-applications

35. Higa, M., Tsuchihashi, T., Abo, M., \& Kakunai, S. (2010). Possibility of Total Hip Arthroplasty Using Shape Memory Alloy. Journal of Biomechanical Science and Engineering, 5, 24-31. https://doi.org/10.1299/jbse.5.24

36. Honório de Carvalho, L., Jr., Machado Soares, L. F., Gonçalves, M. B., Costa, L. L., Costa, L. P., Lessa, R. R., \& Pereira, M. L. (2011). Femoral roll back in total knee arthroplasty: Comparison between prostheses that preserve and sacrifice the posterior cruciate ligament. Revista Brasileira De Ortopedia, 46(4), 417-419. Doi: 10.1016

37. Hu, C.Y. \& Yoon, T.R. (2018). Recent updates for biomaterials used in total hip arthroplasty. Biomaterials Research, 22(33). https://doi.org/10.1186/s40824-018-0144-8

38. Huang, W. M., Ding, Z., Wang, C. C., Wei, J., Zhao, Y., \& Purnawali, H. (2010). Shape memory materials. Materials Today, 13(7), 54-61. https://doi.org/https://doi.org/10.1016/S13697021(10)70128-0

39. Jahan, A., Edwards, K. L., \& Bahraminasab, M. (2016). Case studies of materials selection and design. In Multi-criteria Decision Analysis for Supporting the Selection of Engineering Materials in Product Design (2nd ed., pp. 147-225). Cambridge, MA: Butterworth-Heinemann. Doi: 10.1016

40. Kaivosoja, E., Tiainen, V. M., Takakubo, Y., Rajchel, B., Sobiecki, J., Konttinen, Y. T., \& Takagi, M. (2013). Materials used for hip and knee implants. In Wear of orthopaedic implants and artificial joints. Woodhead Publishing Online. doi:10.1533

41. Kenny, M., Lin-Hua, J., Foster, R., \& Yang, X. B. (2017). Immunological responses to total hip arthroplasty. Journal of Functional Biomaterials, $8(3), \quad 33$. doi:http://dx.doi.org.ezproxy.lib.ou.edu/10.3390/jfb8030033

42. Khoo, B. C. C., Brown, J. K., \& Prince, R. L. (2016). Reconsideration of the effects of age on proximal femur structure: Implications for joint replacement and hip fracture. PLoS One, 11(10) doi:http://dx.doi.org.ezproxy.lib.ou.edu/10.1371/journal.pone.0164949

43. Kirmanidou, Y., Sidira, M., Maria-Eleni Drosou, Bennani, V., Bakopoulou, A., Tsouknidas, A., . . . Michalakis, K. (2016). New ti-alloys and surface modifications to improve the mechanical properties and the biological response to orthopedic and dental implants: A review. BioMed Research International, 2016. doi:http://dx.doi.org/10.1155/2016/2908570

44. Kremers, H.M., Larson, D.R., Crowson, C.S., Kremers, W.K., Washington, R.E., Steiner, C.A., Jiranek, W.A., and Berry, D.J. (2015). Prevalence of Total Hip and Knee Replacement in the United States. The Journal of Bone and Joint Surgery, American Volume, 97(17), 1386-1397. doi: 10.2106/JBJS.N.01141

45. Kunii, T., Mori, Y., Tanaka, H. et al. (2019). Improved Osseointegration of a TiNbSn Alloy with a Low Young's Modulus Treated with Anodic Oxidation. Sci Rep 9, 13985. https://doi.org/10.1038/s41598-019-50581-7

46. Lewallen, E. A., Riester, S. M., Bonin, C. A., Kremers, H. M., Dudakovic, A., Kakar, S., Cohen, R. C., Westendorf, J. J., Lewallen, D. G., \& van Wijnen, A. J. (2015). Biological strategies for improved osseointegration and osteoinduction of porous metal orthopedic implants. Tissue engineering. Part B, Reviews, 21(2), 218-230. https://doi.org/10.1089/ten.TEB.2014.0333

47. Liang-Yu, C., Yu-Wei, C., \& Lai-Chang, Z. (2020). Recent development in beta titanium alloys for biomedical applications. Metals, 10(9), 1139. doi:http://dx.doi.org/10.3390/met10091139

48. Liu, Y., Rath, B., Tingart, M., \& Eschweiler, J. (2020). Role of implants surface modification in osseointegration: A systematic review. Journal of biomedical materials research. Part A, 108(3), 470-484. https://doi.org/10.1002/jbm.a.36829 
49. Lubrizol. What is Crosslinking? What Methods Deliver Different Results?. (2019, June 27). Retrieved October 19, 2020, from https:/www.lubrizol.com/Coatings/Blog/2019/06/What-isCrosslinking

50. Lum, Z. C., Shieh, A. K., \& Dorr, L. D. (2018). Why total knees fail-A modern perspective review. World journal of orthopedics, 9(4), 60-64. https://doi.org/10.5312/wjo.v9.i4.60

51. Luo, C., Wu, X. D., Wan, Y., Liao, J., Cheng, Q., Tian, M., Bai, Z., \& Huang, W. (2020). Femoral Stress Changes after Total Hip Arthroplasty with the Ribbed Prosthesis: A Finite Element Analysis. BioMed research international, 6783936. https://doi.org/10.1155/2020/6783936

52. Ma, C. B., \& Zieve, D. (2018, August 15). Knee joint replacement. Retrieved October 19, 2020, from https://medlineplus.gov/ency/article/002974.htm

53. Ma, R., Tang, S., Tan, H., Lin, W., Wang, Y., Wei, J., Zhao, L., \& Tang, T. (2014). Preparation, characterization, and in vitro osteoblast functions of a nano-hydroxyapatite/polyetheretherketone biocomposite as orthopedic implant material. International journal of nanomedicine, 9, 3949-3961. https://doi.org/10.2147/IJN.S67358

54. Manner, P. W. (2016, April). Knee Replacement Implants. Retrieved October 19, 2020, from https://orthoinfo.aaos.org/en/treatment/knee-replacement-implants/

55. Matweb. Flexural Strength Testing of Plastics. Retrieved November 17, 2020, from $\mathrm{http} / / / \mathrm{www} \cdot \mathrm{matweb} . \mathrm{com} / \mathrm{reference/flexuralstrength.aspx.}$

56. Matweb. Overview of materials for High Density Polyethylene (HDPE), Injection Molded. Retrieved November 17, 2020, from http://www.matweb.com/search/datasheet_print.aspx?matguid=fce23f90005d4fbe8e12a1bce53eb dc8.

57. Merola, M., \& Affatato, S. (2019). Materials for Hip Prostheses: A Review of Wear and Loading Considerations. Materials, 12(3), 495-495. Doi: 10.3390

58. Moraes, M.; Elias, C.; Filho, J.; \& Oliveira, L. (2004). Mechanical Properties of Alumina-Zirconia Composites for Ceramic Abutments. Materials Research-ibero-american Journal of Materials, 7(4), 643-649. Doi: 10.1590/S1516-14392004000400021.

59. Moreno, S. S. (2013). Carbon Reinforced UHMWPE Composites for Orthopaedic Applications. Luleå University of Technology.

60. Mukherjee, K. \& Gupta, S. (2017). Influence of Implant Surface Texture Design on Peri-Acetabular Bone Ingrowth: A Mechanobiology Based Finite Element Analysis. ASME. Journal of Biomechanical Engineering, 139(3), 031006. https://doi.org/10.1115/1.4035369

61. Muratoglu, O. K., Bragdon, C. R., O'Connor, D. O., Jasty, M., \& Harris, W. H. (2001). A Novel Method of Cross-Linking Ultra-HighMolecular-Weight Polyethylene to Improve Wear, Reduce Oxidation, and Retain Mechanical Properties. The Journal of Arthroplasty, 16(2), 149-160. Doi: 10.1054

62. Nabrdalik, M., \& Sobociński, M. (2020). Modeling and computing of stress and strain distribution in UHMW polyethylene elements of chosen artificial human joints. Polish Journal of Chemical Technology, 22(3), 1-8. doi:http://dx.doi.org.ezproxy.lib.ou.edu/10.2478/pjct-2020-0021

63. Panayotov, I.V., Orti, V., Cuisinier, F. et al. Polyetheretherketone (PEEK) for medical applications. J Mater Sci: Mater Med 27, 118 (2016). https://doi.org/10.1007/s10856-016-5731-4

64. Petrini, L., \& Migliavacca, F. (2011). Biomedical Applications of Shape Memory Alloys. Journal of Metallurgy, 2011. https://doi.org/10.1155/2011/501483

65. Pivec, R., Johnson, A. J., Mears, S. C., \& Mont, M. A. (2012). Hip arthroplasty. The Lancet, 380(9855), 1768-77. doi:http://dx.doi.org.ezproxy.lib.ou.edu/10.1016/S0140-6736(12)60607-2

66. Pobloth, A.M., Mersiowsky, M.J., Kliemt, L. et al. (2019). Bioactive coating of zirconia toughened alumina ceramic implants improves cancellous osseointegration. Scientific Reports 9, 16692. https://doi.org/10.1038/s41598-019-53094-5

67. Prasad, K., Bazaka, O., Chua, M., Rochford, M., Fedrick, L., Spoor, J., Symes, R., Tieppo, M., Collins, C., Cao, A., Markwell, D., Ostrikov, K. K., \& Bazaka, K. (2017). Metallic Biomaterials: 
Current Challenges and Opportunities. Materials (Basel, Switzerland), 10(8), 884. https://doi.org/10.3390/ma10080884

68. Ramaraju, H., Solorio, L. D., Bocks, M. L., \& Hollister, S. J. (2020). Degradation properties of a biodegradable shape memory elastomer, poly(glycerol dodecanoate), for soft tissue repair. PLoS One, 15(2) doi:http://dx.doi.org/10.1371/journal.pone.0229112

69. Ribeiro-da-Silva, M., Vasconcelos, D. M., Alencastre, I. S., Oliveira, M. J., Linhares, D., Neves, N., . . Alves, C. J. (2018). Interplay between sympathetic nervous system and inflammation in aseptic loosening of hip joint replacement. Scientific Reports (Nature Publisher Group), 8, 1-11. doi:http://dx.doi.org.ezproxy.lib.ou.edu/10.1038/s41598-018-33360-8

70. Rocha, Rick, Pinheiro, Antônio Luiz Barbosa, \& Villaverde, Antonio Balbin. (2006). Flexural strength of pure Ti, Ni-Cr and Co-Cr alloys submitted to Nd:YAG laser or TIG welding. Brazilian Dental Journal, 17(1), 20-23. https://doi.org/10.1590/S0103-64402006000100005

71. Sergi, R., Bellucci, D., \& Cannillo, V. (2020). A comprehensive review of bioactive glass coatings: State of the art, challenges and future perspectives. Coatings, 10(8), 757. doi:http://dx.doi.org/10.3390/coatings10080757

72. SFB. (2014). Resource Guide. Society for Biomaterials (SFB). https://biomaterials.org/about/resource-guide.

73. So, K., Kanatani, K. T., Kuroda, Y., Nakamura, T., Matsuda, S., \& Akiyama, H. (2012). Good short-term outcome of primary total hip arthroplasty with cementless bioactive glass ceramic bottom-coated implants: 109 hips followed for 3-9 years. Acta orthopaedica, 83(6), 599-603. https://doi.org/10.3109/17453674.2012.736173

74. Sonntag, R., Braun, S., Al-Salehi, L., Reinders, J., Mueller, U., \& Kretzer, J. P. (2017). Threedimensional friction measurement during hip simulation. PloS one, 12(9), e0184043. https://doi.org/10.1371/journal.pone.0184043

75. Teoh, S. H. (2000). Fatigue of biomaterials: a review. International Journal of Fatigue, 22(10), 825837. https://doi.org/https://doi.org/10.1016/S0142-1123(00)00052-9

76. Tilney, R., Burg, M. R., \& Sammut, M. A. (2017). Cobalt cardiomyopathy secondary to hip arthroplasty: An increasingly prevalent problem. Case Reports in Cardiology, 2017, 4. doi:http://dx.doi.org/10.1155/2017/5434571

77. Vahid Golpayegani, M., Sohrabi, A., Biria, M., \& Ansari, G. (2012). Remineralization Effect of Topical NovaMin Versus Sodium Fluoride $(1.1 \%)$ on Caries-Like Lesions in Permanent Teeth. Journal of dentistry (Tehran, Iran), 9(1), 68-75.

78. Werff, H. \& Heisserer, U. (2016). High Performance Ballistic Fibres: Ultra-High Molecular Weight Polyethylene (UHMWPE). In Xiaogang Chen (Ed.), Advanced Fibrous Composite Materials for Ballistic Protection (1st ed., pp.71-108). Woodhead Publishing. Doi: 10.1016/B978-1-78242-4611.00003

79. Westerman, A. P., Moor, A. R., Stone, M. H., \& Stewart, T. D. (2018). Hip Stem Fatigue : The implications of increasing patient mass. Journal of Engineering in Medicine, 232(5), 520-530. Doi: 10.1177

80. Xiong, Y., Du, Q., Wang, Z., \& Wang, A. (2014). Shape memory embracing fixator for Vancouver type B and type C periprosthetic femoral fractures. Acta Orthopaedica Belgica, 80(3), 365-371.

81. Yamane, S., Kyomoto, M., Moro, T., Hashimoto, M., Takatori, Y., Tanaka, S., \& Ishihara, K. (2018). Wear resistance of poly(2-methacryloyloxyethyl phosphorylcholine)-grafted carbon fiber reinforced poly(ether ether ketone) liners against metal and ceramic femoral heads. Journal of biomedical materials research. Part B, Applied biomaterials, 106(3), 1028-1037. https://doi.org/10.1002/jbm.b.33918 